\title{
Natural Sciences and Cultural Sciences: Correlation of Knowledge and Belief*
}

\author{
Ruzana Pskhu \\ Faculty of Humanities and Social Sciences \\ Peoples' Friendship University of Russia \\ 6 Miklukho-Maklaya Street \\ Moscow, Russia, \\ E-mail: r.pskhu@mail.ru
}

\author{
Zurida Murga \\ Faculty of Science \\ Peoples' Friendship University of Russia \\ 6 Miklukho-Maklaya Street \\ Moscow, Russia \\ E-mail: murga_zv@pfur.ru
}

\begin{abstract}
The paper deals with the constitutive elements of creative work in science, which is understood as activities for cognition and explanation of the phenomena of the world and culture, carried out under the certain circumctances. The authors tried to show the main difficulties, which can occur both in the cultural sciences (history of philosophy) and natural sciences (chemistry) in the actual process of cognition of the world and its natural and culture-related realia. The authors follow the classification of sciences proposed by the representatives of Baden school of neo-Kantian philosophy, which differ the natural sciences and cultural sciences.
\end{abstract}

Keywords-cultural sciences; natural sciences; philosophy of science; natural-scientific methods; creativity; knowledge; belief

\section{INTRODUCTION}

Science usually is defined as the process of systemazied image of a part of the reality, which is striving for the discovering the common features of this reality [2]. In this meaning the study of the physical world and its manifestations, especially by using systematic observation and experiment, carried out according to a developed method is in priopity, when we talk about the science. But there are some spheres of reality, which are unavailable for an experiment [3]. We mean the cultural sciences. To say more accurately, there are some spheres of the reality, which need very specialised methods of the investigation, and in this sense the term 'science' needs further elaboration, based on the classification of sciences, proposed by the thinkers of Baden school of neo-Kantian philosophy. For the first time Wilhelm Windelband (1848-1915) and Heinrich Rickert (1863-1936) had put forward the idea, that sciences should be divided into two classes: the sciences of nature and the sciences of culture. The main difference between them is the nature of the objects and methods of cognition: the natural sciences go from the cognition of particularities to discovering of the common universal laws, meanwhile the sciences of culture take into consideration only the concrete and particular realia, which are typical for the human being and specisfic for such a science as a history. The methos, used by the natural sciences, is the generalising method. The

*The publication has been prepared with the support of the "RUDN University Program 5-100". method, used by the sciences of culture, is individualising method. In the first case the main methodological instrument will be an experiment and the result of such a scientific research will be discovering common laws, in the second case instead of expirement as a methodolocial instrument there will be empathy of a scholar and the result of the scientific research will be interpretation. Nevertheless, despite that difference, which makes some scholars refute scientific significance of the humanities, we think that we have the right to talk about the unified sphere of science, which studies both natural and human aspects of the world. And consequently we have possibility to try to find the basic approaches, which will allow us to consider the common problems of a science in total.

Evidently, today the contemporary science has various powerful temtetions. One of these temtetions is the new methods of estimation of the scientific importance and worth of the scientific results. We mean the scientific indexes of quatation. Having put away all difficulties and "good things" of such a criteria of the scientific importance or admittedness (in fact today they are synonims) and having put away all possible critical arguments against it, we would ponder some issues, which are connected with such a scientific criteria.

First, we are interested with two aspects of the scientific creativity, which are in our opinion basic elements of any methodological approach of the scientific investigation. The first one is a knowledge, which is regarded as the complex of common and particular cognitions of a scholar and which allows him not only to orientate himself in the space of the certain science, but also to notice, to arouse and to resolve some special scientific problems. Evidently, that this aspect is common for most scholars and is a general denominator of their scientific communication. The second one is a belief which is regarded in this paper as the complex of presumptions and also as a kind of psychological conviction of a scholar, that the development and resolving of the particular scientific problems is materialized (or is not materialized) with the assistance of some methods which are considered in the certain scientific society as effective ones. We define these notions only for the methodological purpose, and in this paper they should be regarded only in that very specialized meaning. 


\section{NATURAL SCIENCES: HISTORY OF CHEMISTRY}

Nobody will contest that a scholar, who takes as a base of his research knowledge, is very good specialist and professional in a certain sphere of the knowledge. Nevertheless, such a scholar will be unable to resolve a certain type of problems, which need some fundamental revising of the methods, which are used in the current paradigm of the scientific knowledge. Everybody knows these famous various examples in the history of cultural and natural sciences, which contradict the above said thesis about dominant role of knowledge in the process of scientific investigation. Particularly we can remember here the revolution in the Ancient history, which was made by Heinrich Schliemann, who wasn't, as we know, a professional historian. Or we can remember the real story about Niels Bohr, who created in 1913 year the planet construction of atom model, which was discovered by him in the dream.

There are some discoveries in the history of chemistry, which were made due to the new approaches of investigation. For example, the first chemical source of the current was discovered by Alessandro Volta in 1799 in Italy. And the chain of discoveries follows it. In 1800 the Italian scholar Alessandro Volta established, that there is a possibility to choose the metallic plates, immersed into the solution of electrolyte, and in this case the electric current will flow through the wire, which connects them. Volta elaborated the first electric battery, which was the construction, consisted from the twenty pairs of plates, made from different kinds of metal. This battery, which was called "Voltic pile", became the first source of the constant current. The electric current in the galvanic elements always occurs as a result of the chemical reaction, which involves two different metals and the solution of electrolyte, in which they are plunged. For the first time Alessandro Volta gave in his scientific works the indisputable argumentation for the existence the stable connection between the chemical reaction and electricity [4]. As we know, chemistry is a unique science, in which the element of unpredictedness plays a huge role. Another example of it is the story about how Homberg discovered triphenylmethyl radicals. The broken thermometer allowed to discover catalytic impact of sulfate mercury in oxidation of naphthalene to phthalic anhydride and to overcome the last difficulty of the German scholars to the industrial production of dye indigo. The great German chemist Christian Schönbein is said that he discovered in 1846 nitrocellulose and then had investigated its specific characteristics. In 1845 he dropped the oxide on the table and took the dress of his wife to dry it. The material changed its color and Schönbein was interested in it. Having made some investigations he had got the very important scientific results, which are used today in the process of getting dynamite [5].

This period of the history of chemistry is marked by the fact that the chemists for the first time in the history of humanity were be able to synthesize the simple organic compounds. And, as a result of it, the chemical theory of vitality was annihilated. According to this theory of vitality the organic elements can be created only under the influence of the vital power. During the first decades of development and implementation of the structural formulas for the organic compounds there occurred the essential difficulty which was connected with benzene. Benzene is hydrocarbon which consists from six atoms of carbon and six atoms of hydrogen. Solution of this problem was proposed by the great German chemist Friedrich Kekule. Once upon a day, as F. Kekule tells by himself, he was going in the bus and he was dreaming. Suddenly in the dream he saw how atoms were dancing with each other, and unexpectedly the end of the chain of atoms connected with its beginning and he saw the circle of dancing atoms. Immediately Kekule understood that the structural formula of benzene must have the same form benzene ring. He proposed the name 'carbon atom ring' for carbon atoms [6]. This explanation of benzene ring was accepted, and the system of structure formula found its more powerful theoretical foundation. Despite of the usefulness, exactness and accuracy of the structural formulas of Kekule, they were imagined on the flat surface of the paper and didn't reflect the three-dimensional structure of molecule. The last one could explain the optical activity of some substances, which were observed but couldn't be explained at that time. Nevertheless, the question, how the threedimensional nature of molecule could be presented or even imagined, was actual. Because for that period the atomic structure of a substance was only hypothesis and not something more. Nobody hadn't ever seen atoms before and nobody could propose the way, how the atoms could be ordered in the space. The next step was made by the young Dutch chemist (who became later the first Nobel Prize winner in 1901) Jacobus Henricus van't Hoff. He brought up the courageous theoretical proposal, that the four chemical bonds of carbon atom were directed to the four tops of tetrahedron, in the center of which this atom is situated. It is rather interesting that simultaneously the same proposal had been put forward by the French chemist Joseph Achille Le Bel. Tetrahedral atom explained so many things and was so clear, that this theory was accepted by the scientific community rather in a short time [6]. Because there is no more powerful idea than the idea which has come in the proper time. The same interesting story occurred with the discovering of polymer of tetrafluoroethylene or Teflon which became thermostable and chemically inert.

All these examples from the history of chemistry show that the main factors of development of science are not only knowledge, but also a haphazard and the belief of a scholar that the problem under the question could be resolved.

\section{CUlTURAL SCIENCES: HistORY OF ARAB PHILOSOPHY}

In the sphere of philosophical knowledge, we can see that scientific methods of natural sciences (we mean an experiment) work with some reservations (if they could work there at all). The methods used there are called by the neoKantian philosophers as the ideographical methods. Every object of investigation by such an ideographical method has the complex of peculiarities, which makes it a unique element of the world. And as a result, the scientific notions used in the sciences of culture are polysemantic and depend rather on the theoretical background then on the object under 
the consideration. Ultimately the individual phenomena should be described and correlated with the eternal values. And the individual character of it is put forwards as the main one.

The unknown example of revolutionary shift in the history of the particular science is the theory, proposed by the great Russian scholar and philosopher Andrey Smirnov, who resolved the philosophical problem of correlation logic, language and thinking on the base of the Arab culture. Smirnov explains the core of subject-predicate construction as the fact which discovers the inner logic of thinking and its correlation to the reality [7], expressing in the formula " $\mathrm{S}$ is P" (in the case of the Indo-European languages). This formula explains the origin of substantial-attributive metaphysics of the western-European philosophy (eternal striving for the essences). But if we take non-Indo-European language, for example the Arabic language we can see, that this formula " $\mathrm{S}$ is $\mathrm{P}$ " is impossible for that language, because the order of the classical Arabic language excluded its use. The formula of connection of subject and predicate in the Arabic language is the model "P leans S", which presupposes the metaphysics oriented on the process or current of events as systems of things. The connection of subject and predicate doesn't use the verb "to be". In other words, one and the same event can be described in the different languages by principally different means: as things and as processes.

Another example of the ideographical method, used in the cultural sciences, is the investigation of the written heritage of the Iraqi Sufi Muhammad ben 'Abd al-Jabbar anNiffary (X c.). The main problem of its study is the absolute absence of all information about the social and cultural background of this Sufi author. In other words, we haven't any possibility to involve Niffary into the historicalphilosophical context - we have only the 'pure' text. Instead of vast historical-philosophical methodology we can use only linguistic methods of investigation of his texts. We have only Niffary's language and the linguistic methodology of their translation. And the first question arises here is what we should expect as the communicative effect from Niffary's texts, if the only thing we know about them is that they are the dialogue with the God and were not written for people? And the only method of investigation of Niffary's text is investigation of the language structure of them, their lexis and syntax.

The process of such a linguistic investigation of one treatise of Niffary gave very interesting results [8]. The important terminological peculiarity of it is the use of the basic Sufi terms like "fana" (فناء) and 'baqa" (بقاء) only in their verbal form - 'faniya' and 'baqiya'. It seems that for Niffary they don't play very important role as for the Baghdad school of Sufism for example. The main consequence of it is that Niffary at least wasn't an adherent of this school of Junaid, which usually is connected with these terms. But the fact that Niffary uses both terms in pair demonstrates that he knew about them.

The investigation of the Sufi texts based on the study of the linguistic peculiarities of the Arabic language shows some realia, which should be took into consideration by the historian of philosophy: dominant role of the verbal forms, the lack of temporal characteristics of them, the emotional color of semantics for some categories, which lose this peculiarities during their translation into the European languages.

\section{CONCLUSION}

If we define the science as an activity for getting knowledge and explanation of the phenomena of the world, which is realized in the certain conditions, then it is obvious that its main task is arousing and resolving the problems, and as result of it there is the scientific knowledge development. The knowledge allows (a) to put a problem, (b) probably to resolve it and (c) evidently to understand the possibility for it to be resolved on this stage of the science development. The main danger, which faces a scholar is connected with the possibility of the negative reply. Because in the latter case he can stay restricted by the standard methods of resolving problems, which are common for his scientific tradition. To put it in Paul Feyerabend language he will follow "the way of collective resolving of a problem", which is called "guided exchange", "when all participants follow the tradition and agree with those reactions, which are in accordance with its standards" [9]. If this approach is dominant in the science (and we see that it is), and its main task is to create new specialists or the intelligent people, who can work very successfully in the current paradigm of the scientific knowledge, then in fact we can see that the scientific creativity transforms into its stagnation form.

The German thinker Johann Herder defined a culture as the historical level of cultivation of the humankind and related it to the level of development of science and enlightenment. Despite many definitions of it two components of human life become essential, they are material and spiritual components. Most of sciences, chemistry for example, care only the material component of culture. In its turn this material component influences the spiritual and social level of the human society [10]. And we should take into consideration both.

\section{REFERENCES}

[1] Wilhelm Dilthey, Einleitung in die Geisteswissenschaften. Versuch einer Grundlegung für das Studium der Gesellschaft und der Geschichte. Bd. 1. Leipzig, 1883, p. 37.

[2] Dictionary of Philosophical Terms, ed. by V.G. Kuznetsov, Moscow: INFRA-M, 2005, p. 347.

[3] Encarta World English Dictionary. London: Bloomsbury. 1999, p. 1680 .

[4] I. Asimov, A Short History of Chemistry, Moscow: Tsentropolygraph, 2016, pp. 99, 142, 147.

[5] V.M. Gryaznov, Methodology of the Scientific Creativity. Moscow: Izdatelstvo RUDN, 2000, p. 52.

[6] I. Asimov, A Short History of Chemistry, pp. 99, 142, 147.

[7] A.V. Smirnov, Proposition and Predication // Philosophical Journal. Scientific-theoretical journal. V. 9. Moscow: IFRAN, 2016, No.1, pp. 4-5.

[8] Paul Feyerabendt, Science in a Free Society, Moscow: ACT MOSKVA, 2010, p. 78. 
[9] R.V. Pskhu , Translation of Eastern Philosophical Texts in the Context of Historical-Philosophical Problematics. Moscow: RUDN, 2017, p. 292.

[10] I.J. Mittova, A.M. Samoilov, History of Chemistry. In 2 volumes. Vol.1, Moscow: ID, 2012, p. 14. 